\title{
Telomere length and telomerase activity in non-small cell lung cancer prognosis: clinical usefulness of a specific telomere status
}

Tamara Fernández-Marcelo ${ }^{1,4}$, Ana Gómez ${ }^{2,4}$, Irene Pascua ${ }^{1,4}$, Carmen de Juan 1,4, Jacqueline Head 1,4, Florentino Hernando ${ }^{2,4}$, Jose-Ramón Jarabo ${ }^{2,4}$, Joaquín Calatayud ${ }^{2,4}$, Antonio-José Torres-García ${ }^{3,4}$ and Pilar Iniesta ${ }^{14^{*}}$

\begin{abstract}
Background: Considering previous data and the need to incorporate new biomarkers for the prognosis of solid tumours into the clinic, our aim in this work consists of evaluating the potential clinical use of telomeres and telomerase in non-small cell lung cancer (NSCLC).

Methods: Telomere status was established by determination of telomere length using the Terminal Restriction Fragment length method, and telomerase activity by the Telomeric Repeat Amplification Protocol in 142 NSCLCs and their corresponding control samples, obtained from patients submitted to surgery. Group-oriented curves for disease-free survival were calculated according to the Kaplan-Meier method considering telomere length, T/N ratio (telomere length in tumour to control tissue) and telomerase activity.

Results: Overall, tumours had significantly shorter telomeres compared with telomeres in control tissues $(P=0.027)$. More than $80 \%$ of NSCLCs displayed telomerase activity. Regarding prognosis studies, patients whose tumours showed a mean telomere length (MTL) $<7.29 \mathrm{~Kb}$ or T/N ratio $<0.97$ showed a significantly poor clinical evolution ( $P=0.034$ and $P=0.040$, respectively). As result of a Cox multivariate analysis including pathologic state and lymph node dissemination, the MTL and T/N ratio emerged as independent significant prognostic factors.

Conclusions: Telomerase activity was identified as a marker of poor prognosis. The novel finding of this study is the independent prognosis role of a specific telomere status in NSCLC patients. According to our results, telomere function may emerge as a useful molecular tool that allow to select groups of NSCLC patients with different clinical evolution, in order to establish personalized therapy protocols.
\end{abstract}

Keywords: Non-small cell lung cancer, Prognosis, Telomerase, Telomeres

\section{Background}

Lung cancer remains the most common cancer in men worldwide [1] and the identification of subjects at higher risk of cancer progression and recurrence, who would benefit from more specific therapies, is necessary [2]. The risk assessment by the Tumour-Nodes-Metastasis (TNM) staging system is relatively inexact as it only considers tumour characteristics at a particular moment

\footnotetext{
*Correspondence: insepi@ucm.es

'Department of Biochemistry and Molecular Biology II. Faculty of Pharmacy, Complutense University, Sq. Ramón y Cajal s/n (Ciudad Universitaria), Madrid 28040, Spain

${ }^{4}$ Sanitary Research Institute of San Carlos Hospital (IdISSC), Madrid 28040, Spain Full list of author information is available at the end of the article
}

(TNM at diagnosis) [3]. Of fundamental concern is the identification and use of new biomarkers, with high specificity and sensitivity, which will allow the prediction of the disease course and identification of patients who would or would not benefit from specific therapy. In this way, telomere function has been recognized as possible biomarker [2]. However, it has not yet been established a consensus for the use of telomere function in cancer clinical practice.

Telomeres are ribonucleoprotein complexes located at the ends of eukaryotic chromosomes which consist of tandem repeats of a DNA sequence (TTAGGG in all vertebrates) [4]. Among its main functions, telomeres

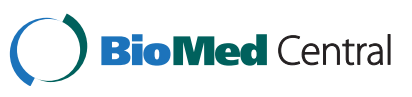


mask double strand break DNA damage signals at the extreme of chromosome and prevent chromosomal fusions [5-8]. In the context of a somatic cell, a significant telomere shortening is monitored by p53 and RB1 and leads to cell death or senescence. However, cells that surpass their normal replicative limit and continue to divide lose all remaining protective telomeric DNA and enter the crisis stage, characterised by massive genomic instability and cell death [9]. At this point, transformed clones emerge and telomerase activation is detectable in the majority of tumour cells and is the main positive power for telomere preservation and elongation [10].

Telomeres and telomerase play a crucial role in human carcinogenesis: the majority of tumours have telomere length alterations which can eventually lead to telomere dysfunction [11]. A disruption of telomere length homeostasis affects telomere structure and leads to genomic instability by generating chromosome end-to end fusions and chromosomal abnormalities [5]. In relation to telomerase, an increase in telomerase activity is often directly correlated with uncontrolled growth of cells, a known hallmark of cancer, and has become a widely acceptable tumour marker and a popular target for anticancer therapeutics [12].

Considering previous data and the need to incorporate new biomarkers for the prognosis of solid tumours into the clinic, our first aim in this work consists of evaluating the potential clinical use of telomeres and telomerase in NSCLC. With this objective, we analyzed both parameters directly implicated in telomere function in a large series of cases of NSCLC. Thus, we identified groups of NSCLC patients with different clinical evolution, independently of the TNM stage tumours, in relation to telomere status (telomere length \& $\mathrm{T} / \mathrm{N}$ ratio).

\section{Methods}

\section{Patients and tissue samples}

One hundred and forty-two primary NSCLCs were obtained from patients who had undergone potentially curative surgery at San Carlos Hospital in Madrid, Spain. Paired non-tumour tissues from the same patient, used as controls, were obtained and microscopically confirmed. After surgical resection, all tissue samples were snap-frozen in liquid nitrogen and stored at $-80{ }^{\circ} \mathrm{C}$ until processed. Cryostat sectioned, H\&E stained samples from each tumour block were examined microscopically by two independent pathologists to confirm the presence of $\geq 80 \%$ tumour cells. All the cases were collected without selection in function of gender, age or tumour stage and no patient had received previous chemotherapy or radiotherapy before diagnosis and entry into this study. Informed consent was obtained from patients prior to investigation and this study was approved by the Ethical Committee of the Hospital.
NSCLCs were staged pathologically using the TNM system $6^{\text {th }}$ Edition [13] and consisted of 86 TNM I tumours, 9 TNM II tumours, 38 TNM IIIA tumours, 5 TNM IIIB tumours and 4 TNM IV tumours. The median follow-up period of patients was 5 years (range, 1-133 months).

\section{Telomere length measurement}

Terminal Restriction Fragment (TRF) length measurement was performed using Telo TTAGGG Telomere Length Assay kit (Roche Applied Science, Germany) as previously described [14]. TRF lengths for tumour and control tissues were determined by comparing the signals relative to a standard molecular weight using Image Gauge software (version 3.46; Fujifilm, Japan). The TRF length ratio was determined as the ratio of the length of tumour tissue TRF and their paired normal tissue TRF ( $\mathrm{T} / \mathrm{N}$ ratio).

\section{Telomerase activity determination}

Telomerase activity was measured using the Telomeric Repeat Amplification protocol (TRAP)-based telomerase polymerase chain reaction (PCR)-enzyme-linked immunosorbent assay (ELISA) kit (Roche Applied Science, Germany), as described earlier [15]. Considering that the cut-off for TRAP-ELISA negativity corresponds to an optical density (OD) $450 \mathrm{~nm}<0.2$, all samples with OD $450 \mathrm{~nm} \geq 0.2$ were considered telomerase positive [15].

\section{Statistical analysis}

Statistical analyses were performed using the SPSS software package (version 19.0 SPSS, Chicago, IL, USA). Differences in telomere length and $\mathrm{T} / \mathrm{N}$ ratio among various groups of patients, discriminated for clinical variables, were analysed by the Student-T and ANOVA tests, or their non-parametric alternatives, Mann-Whitney $\mathrm{U}$ test and Kruskal-Wallis test. $P$-value $<0.05$ was considered statistically significant. The paired samples $T$ test was used for comparing the means of two related variables.

Group-oriented curves for disease-free survival (DFS) were calculated according to the Kaplan-Meier method considering telomere length, $\mathrm{T} / \mathrm{N}$ ratio and telomerase activity. DFS was calculated from the day of surgery until recurrence. The differences in DFS across different groups were compared using the log-rank test. The relative prognostic impact of telomere length, $\mathrm{T} / \mathrm{N}$ ratio and telomerase activity, compared with established prognosis factors, was analysed using Cox multivariate analysis.

Cutoff Finder Web Application [16] was used to determine the cut-off points for prognosis analysis.

\section{Results}

Telomere status and telomerase activity in tissue samples We evaluated telomere length in a total of 284 lung tissue samples: 142 NSCLCs and the corresponding non-tumour 
samples. The mean telomere length (mean \pm standard error) was $6.56 \pm 0.26 \mathrm{~Kb}$ in NSCLCs and $7.00 \pm 0.19 \mathrm{~Kb}$ for non-tumour samples. Overall, tumours had significantly shorter telomeres than matched non-tumour tissues $(P=0.027$; paired $\mathrm{T}$ test $)$. The $\mathrm{T} / \mathrm{N}$ ratio was $0.93 \pm 0.026$. Positive results for telomerase activity were found in 123 (86.6\%) of 142 NSCLCs; 19 tumours $(13.4 \%)$ were telomerase negative.

Telomere status and telomerase activity: correlation with clinical variables of non-small cell lung tumours

As it is showed in the Table 1, there was a statistically significant association between the telomere length and the size of the primary tumour $(\mathrm{T}),(P=0.006$; Kruskal-Wallis test): $\mathrm{T} 1$ tumours had shorter telomeres than both $\mathrm{T} 2$ and T3 tumours. T4 tumours, comprised of a limited number of cases, had a mean telomere length close to T1 tumours. Moreover, there were significant differences between the $\mathrm{T} / \mathrm{N}$ ratio and $\mathrm{T}$ descriptor $(P=0.024$; Kruskal-Wallis test). A significant association was found between the tumour histology and the $\mathrm{T} / \mathrm{N}$ ratio $(P=0.022$; Kruskal-Wallis test), which was significantly diminished in large cell undifferentiated carcinomas compared to squamous cell carcinomas and adenocarcinomas. No association was found neither to telomere length nor $\mathrm{T} / \mathrm{N}$ ratio for the gender, TNM stage, lymph node dissemination $(\mathrm{N})$ and tumour metastasis $(\mathrm{M})$ in NSCLCs (Table 1).

Finally, applying a Chi-square test a statistical association between telomerase activity and the gender $(P=0.034)$, TNM stage $(P=0.042)$, tumour metastasis $(P=0.029)$ and tumour histology $(P=0.019)$ was found (Table 2).

Table 1 Telomere status and clinical variables in non-small cell lung cancers

\begin{tabular}{|c|c|c|c|c|c|}
\hline Variable & $\begin{array}{l}\mathrm{N}^{\circ} \text { of } \\
\text { cases }\end{array}$ & $\begin{array}{l}\text { Telomere length } \\
\text { (Kilobase pairs; } \\
\text { mean } \pm \text { standard error) }\end{array}$ & $\mathrm{P}$ and test statistic & $\begin{array}{l}\text { T/N ratio } \\
\text { (mean } \pm \text { standard error) }\end{array}$ & $\mathrm{P}$ and test statistic \\
\hline Gender & 142 & & & & \\
\hline Female & 12 & $5.49 \pm 0.69$ & 0.274; Mann-Whitney U Test & $0.83 \pm 0.07$ & 0.416; Mann-Whitney U Test \\
\hline Male & 130 & $6.66 \pm 0.28$ & & $0.94 \pm 0.03$ & \\
\hline TNM stage & 142 & & & & \\
\hline 1 & 86 & $6.08 \pm 0.29$ & 0.204; one-way ANOVA & $0.91 \pm 0.03$ & $0.442 ;$ Kruskal-Wallis test \\
\hline$\|$ & 9 & $7.01 \pm 1.37$ & & $0.96 \pm 0.11$ & \\
\hline$\| \mathrm{A}$ & 38 & $7.45 \pm 0.57$ & & $1.00 \pm 0.06$ & \\
\hline$\| I \mid B$ & 5 & $6.15 \pm 1.55$ & & $0.85 \pm 0.18$ & \\
\hline IV & 4 & $7.79 \pm 2.84$ & & $0.80 \pm 0.10$ & \\
\hline Size of the primary tumour, $T$ & 142 & & & & \\
\hline $\mathrm{T} 1$ & 22 & $5.54 \pm 0.52$ & 0.006; Kruskal-Wallis test & $0.93 \pm 0.04$ & 0.024; Kruskal-Wallis test \\
\hline $\mathrm{T} 2$ & 91 & $6.32 \pm 0.30$ & & $0.90 \pm 0.03$ & \\
\hline T3 & 22 & $8.84 \pm 0.84$ & & $1.11 \pm 0.09$ & \\
\hline $\mathrm{T} 4$ & 7 & $5.75 \pm 1.10$ & & $0.79 \pm 0.13$ & \\
\hline Lymph node dissemination, $\mathrm{N}$ & 142 & & & & \\
\hline No & 102 & $6.44 \pm 0.30$ & 0.532; Kruskal-Wallis test & $0.93 \pm 0.03$ & 0.456; Kruskal-Wallis test \\
\hline N1 & 11 & $7.74 \pm 1.22$ & & $1.02 \pm 0.10$ & \\
\hline N2 & 28 & $6.64 \pm 0.62$ & & $0.93 \pm 0.07$ & \\
\hline N3 & 1 & 3.25 & & 0.62 & \\
\hline Tumour metastasis, $\mathrm{M}$ & 142 & & & & \\
\hline Absence, M0 & 138 & $6.52 \pm 0.26$ & 0.848; Mann-Whitney U Test & $0.94 \pm 0.03$ & 0.349; Mann-Whitney U Test \\
\hline Presence, M1 & 4 & $7.79 \pm 2.84$ & & $0.80 \pm 0.10$ & \\
\hline Histology & $141^{\mathrm{a}}$ & & & & \\
\hline $\begin{array}{l}\text { Squamous cell carcinoma } \\
\text { (SCC) }\end{array}$ & 79 & $6.47 \pm 0.33$ & $0.128 ;$ Kruskal-Wallis test & $0.94 \pm 0.03$ & $0.022 ;$ Kruskal-Wallis test \\
\hline Adenocarcinoma (AD) & 56 & $6.93 \pm 0.46$ & & $0.96 \pm 0.04$ & \\
\hline $\begin{array}{l}\text { Large cell undifferentiated } \\
\text { carcinoma (LCUC) }\end{array}$ & 6 & $4.31 \pm 0.89$ & & $0.64 \pm 0.10$ & \\
\hline
\end{tabular}


Table 2 Telomerase activity and clinical variables in non-small cell lung cancers

\begin{tabular}{|c|c|c|c|c|}
\hline \multirow[t]{2}{*}{ Variable } & \multirow[t]{2}{*}{$\mathrm{N}^{\circ}$ of cases } & \multicolumn{2}{|c|}{ Telomerase activity } & \multirow[t]{2}{*}{ P; Chi-square test } \\
\hline & & Negative & Positive & \\
\hline Gender & 142 & & & \\
\hline Female & 12 & 4 & 8 & \multirow{2}{*}{0.034} \\
\hline Male & 130 & 15 & 115 & \\
\hline TNM stage & 142 & & & \multirow{6}{*}{0.042} \\
\hline I & 86 & 12 & 74 & \\
\hline$\|$ & 9 & 1 & 8 & \\
\hline$\| I I A$ & 38 & 2 & 36 & \\
\hline$\| I \mid B$ & 5 & 2 & 3 & \\
\hline IV & 4 & 2 & 2 & \\
\hline Size of the primary tumour, $T$ & 142 & & & \multirow{5}{*}{0.126} \\
\hline $\mathrm{T} 1$ & 22 & 3 & 19 & \\
\hline $\mathrm{T} 2$ & 91 & 11 & 80 & \\
\hline T3 & 22 & 2 & 20 & \\
\hline $\mathrm{T} 4$ & 7 & 3 & 4 & \\
\hline Lymph node dissemination, $\mathrm{N}$ & 142 & & & \\
\hline NO & 102 & 13 & 89 & \multirow{4}{*}{0.838} \\
\hline N1 & 11 & 1 & 10 & \\
\hline N2 & 28 & 5 & 23 & \\
\hline N3 & 1 & 0 & 1 & \\
\hline Tumour metastasis, $\mathrm{M}$ & 142 & & & \multirow{3}{*}{0.029} \\
\hline Absence, M0 & 138 & 17 & 121 & \\
\hline Presence, M1 & 4 & 2 & 2 & \\
\hline Histology & $141^{\mathrm{a}}$ & & & \\
\hline Squamous cell carcinoma (SCC) & 79 & 8 & 71 & \multirow{3}{*}{0.019} \\
\hline Adenocarcinoma (AD) & 56 & 7 & 49 & \\
\hline Large cell undifferentiated carcinoma (LCUC) & 6 & 3 & 3 & \\
\hline
\end{tabular}

aissing data

Considering our studied population, no significant differences were found between the telomere lengths of negative and positive telomerase activity tumours $(P=0.549$; Mann-Whitney $\mathrm{U}$ test). The mean telomere length (mean \pm typical error) was $7.16 \pm 0.87 \mathrm{~Kb}$ in negative telomerase activity tumours and $6.47 \pm 0.27 \mathrm{~Kb}$, for positive telomerase activity tumours. The range of tumour telomere lengths between the groups defined by telomerase activity was similar: $1.51-16.49 \mathrm{~Kb}$ for negative telomerase activity tumours and $1.42-17.50 \mathrm{~Kb}$ for positive telomerase activity tumours.

\section{Telomere status, telomerase activity and NSCLC patients prognosis}

Prognosis studies were developed to assess the impact on clinical course of telomere status (telomere length \& $\mathrm{T} / \mathrm{N}$ ratio) and telomerase activity in patients with resected NSCLCs. Only patients who had undergone potentially curative surgery and who did not die during the postoperatory period were considered in these studies, as established in the literature. A total of 125 cases were considered. First, patients were stratified into two groups according to the mean telomere length using the Cutoff Finder Web Application [16]: the group of patients who had tumours with a mean telomere length $<7.29 \mathrm{~Kb}$ presented a significantly poor clinical evolution, compared to the group whose mean tumour telomere length was $>7.29$ $\mathrm{Kb}(P=0.034 ;$ Log-Rank. Fig. 1a). Forty percent $(32 / 80)$ of patients included in the first group relapsed during the follow-up period, compared with the second group where $20 \%$ of patients $(9 / 45)$ experienced a relapse. The KaplanMeier curves show this relationship between the telomere length and disease-free survival (Fig. 1a).

Next, using the Cutoff Finder Web Application [16] the best prognostic cut-off for the $\mathrm{T} / \mathrm{N}$ ratio was set in 0.97: patients who had tumours with a $\mathrm{T} / \mathrm{N}$ ratio 

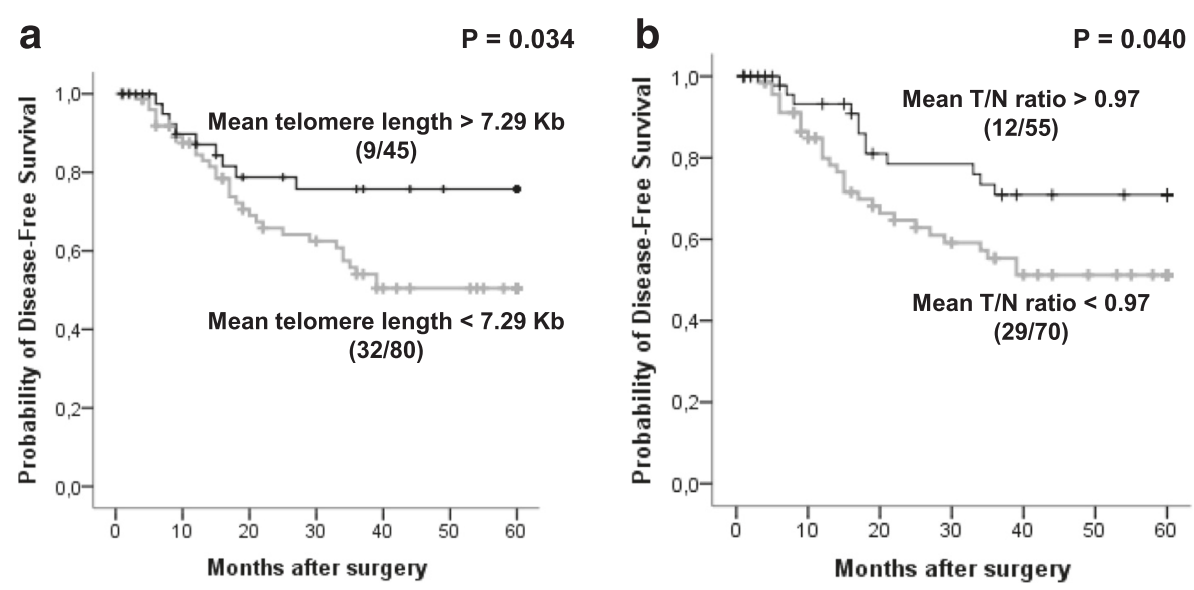

Fig. 1 Kaplan-Meier plots of Disease Free Survival (DFS) considering telomere status. Kaplan-Meier survival curves in relation to telomere length (a) and T/N ratio (b) in non-small cell lung cancer. Numbers in brackets represent cases with tumour recurrence and crosses indicate censored data

$<0.97$ had a significantly shorter disease-free survival compared with patients who had a bigger $\mathrm{T} / \mathrm{N}$ ratio ( $P=0.040$; Log-Rank. Fig. 1b).

As result of a Cox multivariate analysis, including TNM stage and lymph node dissemination (only these factors had prognostic relevance in the univariate analysis, $P<0.001$ ), telomere length emerged as an independent significant prognostic factor (Table 3A and B). In both cases, the patients with a mean telomere length $<7.29 \mathrm{~Kb}$ were at increased risk for recurrence. Also using the Cox multivariate analysis, a mean $\mathrm{T} / \mathrm{N}$ ratio $<0.97$ was found to be a prognostic factor independent of the TNM stage $(P=0.041)$ (Table $4 \mathrm{~A}$ and $\mathrm{B})$.

Finally, telomerase activity discriminated between two groups of patients: the absence of telomerase activity in the tumour conferred a better clinical evolution ( $P=0.039 ;$ Log-Rank. Fig. 2). In multivariate analysis, a clear trend towards a potential role for telomerase activity as an independent prognostic factor of the TNM stage $(P=0.089)$ and lymph node dissemination $(P=0.101)$ was found.

Table 3 Multivariate Cox Regression Analysis considering TNM stage (A), lymph node dissemination (B) and telomere length for 125 patients with non-small cell lung cancer

\begin{tabular}{lcc}
\hline \multicolumn{1}{c}{ (A) Variable } & HR (95 \% Cl) & P \\
\hline $\begin{array}{l}\text { Mean telomere length in } \\
\text { NSCLCs }<7.29 ~ K b\end{array}$ & $2.70(1.27-5.76)$ & 0.010 \\
TNM stage, I vs. II or IIIA & $0.34(0.17-0.67)$ & 0.002 \\
\hline \multicolumn{1}{c}{ (B) Variable } & RR (95\% Cl) & $\mathbf{P}$ \\
\hline $\begin{array}{l}\text { Mean telomere length in } \\
\text { NSCLCs <7.29 Kb }\end{array}$ & $2.26(1.07-4.76)$ & 0.032 \\
$\begin{array}{l}\text { Lymph node dissemination (N), } \\
\text { negative (N0) vs. positive (N1 or N2) }\end{array}$ & $0.41(0.19-0.88)$ & 0.021 \\
\hline
\end{tabular}

$H R$ hazard ratio, $\mathrm{Cl}$ confidence interval

\section{Discussion}

Malignant cells, in general, have shorter telomeres than their normal counterparts [17] as a reflection of their extended proliferation. In fact, diseases of high cellular turnover are associated with telomere shortening, telomere dysfunction and cancer predisposition [18]. In bronchial carcinogenesis, telomere shortening is an early event [19] and previous results [20] including those of our research group [15] show a significant telomere shortening in NSCLCs in relation to the control tissues. In other tumour types, telomere attrition has been also demonstrated [21]. Therefore, the results shown in this paper, as with the previous data, reflect differences between the mean telomere length in NSCLCs and the non-tumour tissues, which are validated by a $\mathrm{T} / \mathrm{N}$ ratio $<1$.

Progressive telomere erosion is countered with telomerase, which is expressed in the vast majority of human cancers [12]. Our data indicated more than $80 \%$ of NSCLCs analysed expressed telomerase activity, in agreement with previously published works [15], without significant differences in the mean telomere length values between telomerase positive and negative tumours. Although telomerase activation can be an early event in cancer, it is not necessary for cancer initiation. However,

Table 4 Multivariate Cox Regression Analysis considering TNM stage (A), lymph node dissemination (B) and T/N ratio for 125 patients with non-small cell lung cancer

\begin{tabular}{lcc}
\hline \multicolumn{1}{c}{ (A) Variable } & HR $(\mathbf{9 5} \%$ Cl) & P \\
\hline Mean T/N ratio $<0.97$ & $2.02(1.03-3.97)$ & 0.041 \\
TNM stage, I vs. II or IIIA & $0.42(0.21-0.81)$ & 0.010 \\
\hline \multicolumn{1}{c}{ (B) Variable } & RR $(\mathbf{9 5} \%$ Cl) & $\mathbf{P}$ \\
\hline Mean T/N ratio $<0.97$ & $1.93(0.98-3.81)$ & 0.058 \\
Lymph node dissemination (N), & $0.44(0.21-0.95)$ & 0.036 \\
negative (N0) vs. positive (N1 or N2) & & \\
\hline
\end{tabular}

$H R$ hazard ratio, $\mathrm{Cl}$ confidence interval 


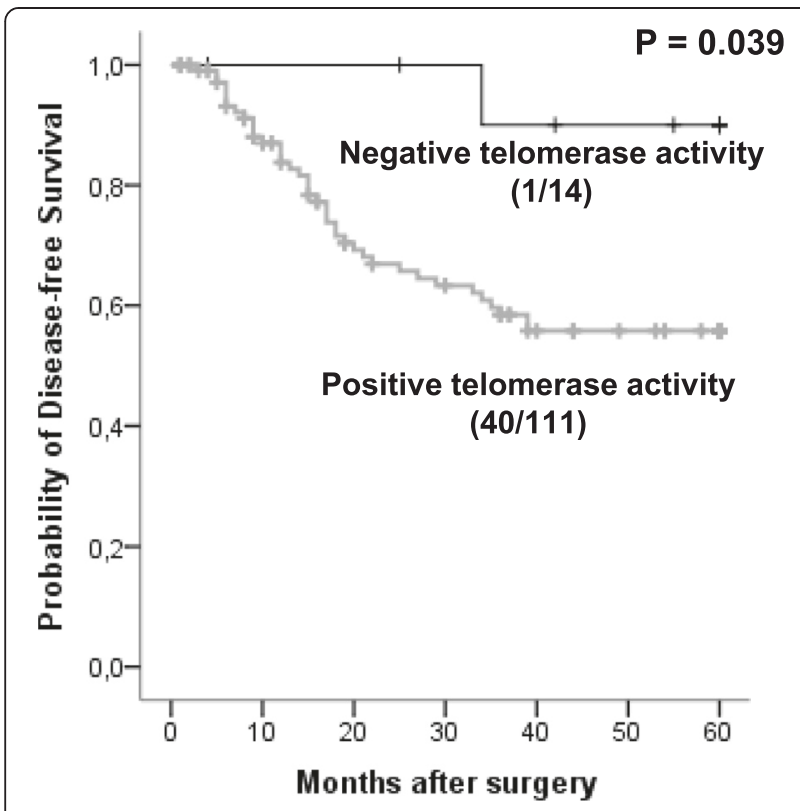

Fig. 2 Kaplan-Meier plots of Disease Free Survival (DFS) considering telomerase activity. Kaplan-Meier survival curves in relation to telomerase activity. Numbers in brackets represent cases with tumour recurrence and crosses indicate censored data

telomerase can stimulate tumour progression by ensuring maintenance of telomeres above a critically short length, thus preventing induction of cellular senescence or apoptosis [22]. Appart from telomerase, possible implication of Alternative Lengthening of Telomeres (ALT) mechanisms in telomere elongation of telomerase-negative tumours should be considered. However, the ALT phenotype was not investigated in our research work considering the limited role of this mechanism in lung cancer. In fact, the ALT phenotype is common in tumours such as osteosarcomas, undifferentiated pleomorphic sarcomas, leiomyosarcomas, astrocytic tumours grades 2 and 3, and pancreatic neuroendocrine tumours [11, 23]. However, ALT is a very infrequent mechanism in the most common cancer types, carcinomas, which are derived from epithelia [24]. Heaphy et al. assessed the ALT phenotype in 6110 primary tumours from 94 different cancer subtypes and this mechanism was not detected in most lung carcinoma subtypes, only individual cases of small cell (2\%) and large cell lung carcinomas (3\%) were observed. ALT-positive cells are characterized by striking telomere length heterogeneity [11]. In the present work, it is noteworthy that the maximum value in telomere length for telomerase positive and negative lung tumours is close to that defined in the normal human population: the length of telomeres is heterogeneous, ranging between 5 and $15 \mathrm{~Kb}$ [25]. Therefore, very long telomeres, other feature of ALT, were not detected.
The role of telomeres in the initiation and progression of carcinogenesis has been widely recognized [11]; thus, its relation with clinical variables, in conjunction with others molecular markers, could represent a therapeutic opportunity for cancer patients. Currently, except for recent developments in relation to the involvement of EGFR mutational status [26], limited advances in the detection of molecular targets in lung cancer were obtained. For NSCLCs, the statistical association between the tumour size ( $\mathrm{T}$ descriptor) and telomere status is in line with previously published data [15]: the highest degree of telomere shortening is detected for tumours that grow into the area of mediastinum or cancers in which a malignant pleural effusion is reached (T4).

An altered telomere length in cancer cells could give the ability to metastasize and cause recurrent disease and, in consequence, be a predictor of clinical outcome. Previous works concluded that careful assessment of telomere length or its proxies, such as DNA content, will be part of novel risk assessment and prognostic modalities for patients [2]. More recently, in glioblastoma multiform tumours, telomeres were always shorter when compared with normal brain tissue, and together with telomerase activity seem to be associated with malignancy and poor outcome [27]. In lung cancer, previous studies have evaluated whether telomere length could represent a risk factor or a prognostic marker, however most of them are contradictory: for NSCLCs patients both shorter and longer telomeres has been associated with decreased overall survival [28]. Results obtained in the present work indicate that the worst prognosis is seen in patients whose mean tumour telomere length is lower than $7.29 \mathrm{~Kb}$ or when almost any degree of telomere shortening is reached in tumours cells. Both parameters related to telomere status proved to be independent of other known prognostic factors. However, we also demonstrated in colorectal cancer that telomere attrition conferred good clinical evolution. Therefore, cancer prognosis associated to telomere status could be dependent on the tumour type [14]. A deficiency in the function of senescence and cell death pathways [29], or other molecules related to telomere maintenance and genome instability [30] could explain the unfavorable prognosis for post-surgical patients with NSCLCs and short telomeres. Genetic context underlying telomere status, which confers the different clinical outcome, must be carefully considered.

Recently, two works have demonstrated that leukocyte relative telomere length in peripheral blood is an independent prognostic marker in glioma and gastric cancer patients, being shorter telomeres associated with the worst clinical evolution of patients [31, 32]. Telomere analyses in leukocytes from peripheral blood could be a useful biomarker to improve the prognosis prediction in cancer 
patients. However, previously it is necessary corroborate and validate correlations between telomere status in blood cells and tumours through prospective studies. This is a field that needs to be investigated in lung cancer.

Finally, the presence of telomerase activity in NSCLCs conferred the worst outcome, confirming previous results [15]. Telomerase activity or hTERT expression has proved to be a marker of malignancy. Furthermore, its diagnostic utility is being demonstrated [11].

\section{Conclusions}

The novel finding of this study is the independent prognosis role of a specific telomere status in NSCLC patients. Moreover, telomerase activity is confirmed as a prognostic marker in NSCLC. According to results from the present work, telomere function may emerge as a useful molecular tool that allow to select groups of NSCLC patients with different clinical evolution, in order to establish personalized therapy protocols.

\section{Competing interests}

The authors declare that they have no competing interests.

\section{Authors' contributions}

TFM carried out most of the molecular studies, the statistical analysis, participated in interpretation of data, and was involved in drafting the manuscript. IP, CDJ and JH participated in molecular analysis and interpretation of data. AG, FH, JRJ and JC participated in analysis and interpretation of data, as well as in advice on possible clinical implications of results from this work. AJTG has been involved in revising the manuscript. PI carried out the design and coordination of the study, and drafted the manuscript. All authors have read and approved the final manuscript.

\section{Acknowledgments}

This work was supported by grants from Fundación de Investigación Médica Mutua Madrileña, Neumomadrid and Santander-UCM.

\section{Author details}

${ }^{1}$ Department of Biochemistry and Molecular Biology II. Faculty of Pharmacy, Complutense University, Sq. Ramón y Cajal s/n (Ciudad Universitaria), Madrid 28040, Spain. ${ }^{2}$ Service of Thoracic Surgery, San Carlos Hospital, Madrid 28040, Spain. ${ }^{3}$ Service of General Surgery and Digestive Tract, San Carlos Hospital, Madrid 28040, Spain. ${ }^{4}$ Sanitary Research Institute of San Carlos Hospital (IdISSC), Madrid 28040, Spain.

Received: 23 April 2015 Accepted: 21 July 2015

Published online: 07 August 2015

\section{References}

1. Ferlay J, Soerjomataram I, Ervik M, Dikshit R, Eser S, Mathers C, et al. GLOBOCAN 2012 v1.0, Cancer Incidence and Mortality Worldwide: IARC Cancer Base No. 11. Lyon, France: International Agency for Research on Cancer; 2013. Available on http://globocan.iarc.fr

2. Bisoffi M, Heaphy CM, Griffith JK. Telomeres: prognostic markers for solid tumors. Int J Cancer. 2006;119:2255-60.

3. Burke HB. Outcome prediction and the future of the TNM staging system. J Natl Cancer Inst. 2004;96:1408-9.

4. de Lange T. Shelterin: the protein complex that shapes and safeguards human telomeres. Genes Dev. 2005:19:2100-10.

5. de Lange T. How telomeres solve the end-protection problem. Science. 2009:326:948-52

6. O'Sullivan RJ, Karlseder J. Telomeres: protecting chromosomes against genome instability. Nat Rev Mol Cell Biol. 2010;11:171-81.

7. de Lange T. Protection of mammalian telomeres. Oncogene. 2002;21:532-40.
8. Donate $L E$, Blasco MA. Telomeres in cancer and ageing. Phil Trans R Soc B. 2011;366:76-84.

9. Verdun RE, Karlseder J. Replication and protection of telomeres. Nature. 2007:447:924-31.

10. Svenson U, Roos G. Telomere length as a biological marker in malignancy. Biochim Biophys Acta. 2009;1792:317-23.

11. Heaphy CM, Meeker AK. The potential utility of telomere-related markers for cancer diagnosis. J Cell Mol Med. 2011;15:1227-38.

12. Ruden M, Puri N. Novel anticancer therapeutics targeting telomerase. Cancer Treat Rev. 2013:39(5):444-56.

13. Mountain CF. Revisions in the International System for Staging Lung Cancer. Chest. 1997;111:1710-7.

14. Garcia-Aranda C, de Juan C, Diaz-Lopez A, Sanchez-Pernaute A, Torres A,

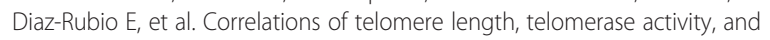
telomeric-repeat binding factor 1 expression in colorectal carcinoma. Cancer. 2006;106:541-51.

15. Frías C, García-Aranda C, de Juan C, Morán A, Ortega P, Gómez A, et al. Telomere shortening is associated with poor prognosis and telomerase activity correlates with DNA repair impairment in non-small cell lung cancer. Lung Cancer. 2008;60:416-25.

16. Budczies J, Klauschen F, Sinn BV, Győrffy B, Schmitt WD, Darb-Esfahani S, et al. Cutoff Finder: a comprehensive and straightforward Web application enabling rapid biomarker cutoff optimization. Plos ONE. 2012;7:e51862.

17. Hackett JA, Greider CW. Balancing instability: dual roles for telomerase and telomere dysfunction in tumorigenesis. Oncogene. 2002;21:619-26.

18. O'Sullivan JN, Bronner MP, Brentnall TA, Finley JC, Shen W, Emerson S, et al. Chromosomal instability in ulcerative colitis is related to telomere shortening. Nat Genet. 2002;32:280-4.

19. Lantuejoul S, Soria JC, Morat L, Lorimier P, Moro-Sibilot D, Sabatier L, et al. Telomere shortening and telomerase reverse transcriptase expression in preinvasive bronchial lesions. Clin Cancer Res. 2005;11:2074-82.

20. Hsu CP, Miaw J, Shai Sen E, Chen CY. Correlation between telomerase expression and terminal restriction fragment length ratio in non-small cell lung cancer-an adjusted measurement and its clinical significance. Eur J Cardio-Thorac Surg. 2004;26:425-31.

21. Rha SY, Park KH, Kim TS, Yoo NC, Yang WI, Roh JK, et al. Changes of telomerase and telomere lengths in paired normal and cancer tissues of breast. Int J Oncol. 1999;15:839-45.

22. Jesus BB, Blasco MA. Telomerase at the intersection of cancer and aging. Trends In Genetics. 2013;29:513-20.

23. Henson JD, Reddel RR. Assaying and investigating Alternative Lengthening of Telomeres activity in human cells and cancer. FEBS Lett. 2010;584:3800-11.

24. Shay JW, Reddel RR, Wright WE. Cancer and telomeres-an ALTernative to telomerase. Science. 2012;336:1388-90.

25. Samassekou O, Gadji M, Drouin R, Yan J. Sizing the ends: normal length of human telomeres. Ann Anat. 2010;192(5):284-91.

26. Wei B, Yang K, Zhao J, Chang Y, Ma Z, Dong B, et al. Quantification of EGFR mutations in primary and metastatic tumors in non-small cell lung cancer. J Exp \& Clin Cancer Res. 2014;33:5. doi:10.1186/1756-9966-33-5.

27. Torre DL, Aguennouz M, Conti A, Giusa M, Raffa G, Abbritti RV, et al. Potential clinical role of telomere length in human glioblastoma. Transl Med UniSa. 2011;1:243-70.

28. Gansner JM, Rosas IO. Telomeres in lung disease. Transl Res. 2013;162:343-52.

29. Fernández-Marcelo T, Morán A, de Juan C, Pascua I, Head J, Gómez A, et al. Differential expression of senescence and cell death factors in non-small cell lung and colorectal tumors showing telomere attrition. Oncology. 2012:82:153-64.

30. Fernández-Marcelo T, Frías C, Pascua I, de Juan C, Head J, Gómez A, et al. Poly(ADP-ribose) polymerase 3 (PARP3), a potential repressor of telomerase activity. J Exp \& Clin Cancer Res. 2014;33:19. doi:10.1186/1756-9966-33-19.

31. Chen Y, Wu Y, Huang X, Qu P, Li G, Jin T, et al. Leukocyte telomere length: a novel biomarker to predict the prognosis of glioma patients. J Cancer Res Clin Oncol. 2015. doi:10.1007/s00432-015-1938-X.

32. Qu F, Li R, He X, Li Q, Xie S, Gong L, et al. Short telomere length in peripheral blood leukocyte predicts poor prognosis and indicates an immunosuppressive phenotype in gastric cancer patients. Mol Oncol. 2015;9:727-39. 